\title{
Neighbor Sum Distinguishing Index
}

\author{
Evelyne Flandrin • Antoni Marczyk • \\ Jakub Przybyło • Jean-François Saclé • \\ Mariusz Woźniak
}

\begin{abstract}
We consider proper edge colorings of a graph $G$ using colors of the set $\{1, \ldots, k\}$. Such a coloring is called neighbor sum distinguishing if for any pair of adjacent vertices $x$ and $y$ the sum of colors taken on the edges incident to $x$ is different from the sum of colors taken on the edges incident to $y$. The smallest value of $k$ in such a coloring of $G$ is denoted by $\operatorname{ndi}_{\Sigma}(G)$. In the paper we conjecture that for any connected graph $G \neq C_{5}$ of order $n \geq 3$ we have $\operatorname{ndi}_{\Sigma}(G) \leq \Delta(G)+2$. We prove this conjecture for several classes of graphs. We also show that $\operatorname{ndi}_{\Sigma}(G) \leq 7 \Delta(G) / 2$ for any graph $G$ with $\Delta(G) \geq 2$ and $\operatorname{ndi}_{\Sigma}(G) \leq 8$ if $G$ is cubic.
\end{abstract}

Keywords Proper edge coloring $\cdot$ Neighbor-distinguishing index $\cdot$ Neighbor sum distinguishing coloring $\cdot$ Chromatic index

\section{Mathematics Subject Classification $\quad 05 \mathrm{C} 15$}

\section{Introduction and Terminology}

Let $G$ be a finite undirected simple graph. We denote by $V=V(G)$ its vertex-set and by $E=E(G)$ its set of edges.

The work of the authors from AGH University of Science and Technology was partially supported by the Polish Ministry of Science and Higher Education.

E. Flandrin · J.-F. Saclé

L R I, UMR 8623, Bât. 490, Université de Paris-Sud, 91405 Orsay, France

A. Marczyk $(\varangle) \cdot$ J. Przybyło · M. Woźniak

AGH University of Science and Technology, al. A. Mickiewicza 30, 30-059 Krakow, Poland e-mail: marczyk@agh.edu.pl 
Let $C$ be a finite set of colors and let $f: E(G) \rightarrow C$ be a proper edge coloring of $G$. The color set of a vertex $v \in V(G)$ with respect to $f$ is the set $S(v)$ of colors of edges incident to $v$. The coloring $f$ is neighbor-distinguishing if it distinguishes any two adjacent vertices by their color sets, i.e., $S(u) \neq S(v)$ whenever $u, v \in V(G)$ and $u v \in E(G)$.

As usually, we are interested in the smallest number of colors in a neighbor-distinguishing coloring of $G$. This number will be called the neighbor-distinguishing index of the graph $G$ and will be denoted by $\operatorname{ndi}(G)$.

Evidently, when searching for the neighbor-distinguishing index it is sufficient to restrict our attention to connected graphs. Observe also, that $G=K_{2}$ does not have any neighbor-distinguishing coloring. So, we shall consider only connected graphs with at least three vertices.

This invariant has been introduced by Zhang et al. [8]. It is easy to see that $\operatorname{ndi}\left(C_{5}\right)=$ 5 and in [8] it is conjectured that $\operatorname{ndi}(G) \leq \Delta(G)+2$ for any connected graph $G \neq C_{5}$ on $n \geq 3$ vertices. The conjecture has been confirmed by Balister et al. [3] for bipartite graphs and for graphs $G$ with $\Delta(G)=3$. Edwards et al. [5] have shown even that $\operatorname{ndi}(G) \leq \Delta(G)+1$ if $G$ is bipartite, planar, and of maximum degree $\Delta(G) \geq 12$.

The aim of this paper is to study a similar invariant. A proper $[k]$-edge coloring of a simple graph $G$ is an assignment $v$ of integers from the set $[k]=\{1, \ldots, k\}$ to the edges of $G$. In other words, we put $C=[k]$. In this case, we are allowed to consider the sum of colors at each vertex i.e., the number

$$
w(x)=\sum_{e \ni x} v(e)
$$

A proper $[k]$-edge coloring of $G$ is called neighbor sum distinguishing (nsd for short) if

$$
w(x) \neq w(y)
$$

whenever $x y \in E(G)$. In other words, the vertex coloring $w$ induced by $f$ in the above described way is required to be proper. The corresponding invariant, i.e., the minimum value of $k$ for such an nsd proper [k]-edge coloring of $G$, is called the neighbor sum distinguishing index of $G$ and denoted by $\operatorname{ndi}_{\Sigma}(G)$.

Clearly, if the sums of colors in $S(x)$ and $S(y)$ are distinct then the sets $S(x)$ and $S(y)$ are also distinct. So, for every connected graph $G$ of order $n \geq 3$ we have

$$
\Delta(G) \leq \chi^{\prime}(G) \leq \operatorname{ndi}(G) \leq \operatorname{ndi}_{\Sigma}(G)
$$

where $\chi^{\prime}(G)$ denotes the chromatic indeks of $G$.

We propose the following conjecture.

Conjecture 1 If $G$ is a connected graph on at least 3 vertices and $G \neq C_{5}$, then $\Delta(G) \leq \operatorname{ndi}_{\Sigma}(G) \leq \Delta(G)+2$.

It is also worth mentioning here that our reasonings correspond with the recent study (see [7]) of [ $k]$-edge colorings distinguishing all (not only adjacent) vertices 
by their respective sums. Such a coloring is called vertex sum distinguishing and the corresponding parameter is denoted by $\operatorname{vdi}_{\Sigma}(G)$.

Clearly, we have $\operatorname{ndi}_{\Sigma}(G) \leq \operatorname{vdi}_{\Sigma}(G)$ for every connected graph $G$ of order $n \geq 3$.

In the sequel we denote by $K_{n, n}^{\prime}$ the graph obtained from the complete bipartite graph $K_{n, n}$ by erasing a perfect matching and by $P_{m}$ the path of order $m$. We use Bondy and Murty's book [2] for terminology and notation not defined here.

\section{Paths, Cycles, Complete Graphs and Complete Bipartite Graphs}

The proofs of the following two propositions are left to the reader.

Proposition $2 \operatorname{ndi}_{\Sigma}\left(P_{3}\right)=2, \operatorname{ndi}_{\Sigma}\left(P_{m}\right)=3, m \geq 4$.

Proposition $3 \operatorname{ndi}_{\Sigma}\left(C_{5}\right)=5, \operatorname{ndi}_{\Sigma}\left(C_{m}\right)=3$ if $m \equiv 0(\bmod 3)$ and $\operatorname{ndi}_{\Sigma}\left(C_{m}\right)=4$ otherwise.

Observe now that if $G$ is a complete graph then $\operatorname{ndi}_{\Sigma}(G)=\operatorname{vdi}_{\Sigma}(G)$. Hence, the following proposition is an easy collorary of an analogous result of [7].

Proposition 4 For every $n \geq 3$

$$
\operatorname{ndi}_{\Sigma}\left(K_{n}\right)= \begin{cases}n ; & \text { if } n \text { is odd } \\ n+1 ; & \text { if } n \text { is even }\end{cases}
$$

Proposition $5 \operatorname{ndi}_{\Sigma}\left(K_{n, n}\right)=n+2$.

Proof It is shown in [7] that $\operatorname{vdi}_{\Sigma}\left(K_{n, n}\right)=n+2$, hence $\operatorname{ndi}_{\Sigma}\left(K_{n, n}\right) \leq n+2$. Obviously, $\operatorname{ndi}_{\Sigma}\left(K_{n, n}\right) \geq n+1$, because there are in $K_{n, n}$ at least two adjacent vertices of maximum degree. Suppose $v$ is a proper nsd $[n+1]$-edge coloring of $K_{n, n}$. Then at least one color class, say $a$, does not induce a perfect matching in this graph. Therefore, there are two adjacent vertices $x$ and $y$, such that $a$ is lacking in the set of colors of edges incident to $x$ and in the set of colors of edges incident to $y$. Thus $w(x)=w(y)$, a contradiction.

Any proper coloring of $K_{n, p}(n \geq 2, p<n)$ using colors of [ $\left.n\right]$ is an nsd [ $n$ ]-edge coloring. So we have the following observation.

Proposition $6 \operatorname{ndi}_{\Sigma}\left(K_{n, p}\right)=n$, if $n \geq 2$ and $p<n$.

\section{An Upper Bound}

Theorem 7 For each connected graph $G$ with maximum degree $\Delta \geq 2$, we have

$$
\operatorname{ndi}_{\Sigma}(G) \leq\left\lceil\frac{7 \Delta-4}{2}\right\rceil
$$


Proof We will use induction with respect to $m=\|G\|$ (the size of $G$ ), to prove the existence of the desired coloring. Since the assertion is obvious for $m=2$, we may assume that $m \geq 3$ and that an appropriate coloring does exist for each graph of size smaller than $m$. Fix a connected graph $G$ with $\|G\|=m$ and $\Delta \geq 2$. Choose any vertex $b$ of degree $\geq 2$ and two edges incident to it, say $a b$ and $b c$. We may assume that we can choose $a, b$ and $c$ in such a way that the vertices $a$ and $c$ are not adjacent, because otherwise $G$ would be a complete graph and for these graphs the theorem is true (see Proposition 4). Let $H$ be the graph obtained from $G$ by removing the edges $a b$ and $b c$. By the induction hypothesis, there exist neighbor sum distinguishing $\left[\left\lceil\frac{7 \Delta-4}{2}\right\rceil\right]$-edge colorings for each of the components of $H$ with at least two edges. Use 1 to color the edges of the remaining components, which are simply isolated edges and vertices. We shall extend these colorings to the desired coloring of $G$.

Assume first that the sets $N_{H}(a), N_{H}(b), N_{H}(c)$ are all nonempty. Let $a_{1}, \ldots, a_{p}$ be the neighbors of $a$ in $H$, let $b_{1}, \ldots, b_{q}$ be the neighbors of $b$ in $H$, and let $c_{1}, \ldots, c_{r}$ be the neighbors of $c$ in $H(p, r \leq \Delta-1, q \leq \Delta-2)$. We shall first focus on the edge $a b$, and estimate how many colors we may use to paint it, and still be able to extend our coloring to the desired one. We surely cannot use the colors of at most $(\Delta-1)+(\Delta-2)$ (already painted) edges adjacent to $a b$. Note subsequently that while choosing the color of $a b$, we fix the sum at $a$, and since the sums of $a_{1}, \ldots, a_{p}$ are already determined, we may have to exclude additional (at most) $\Delta-1$ colors from the list of possible choices. Of the remaining ones, at most one may moreover give us the same sums at $b$ and $c$ (regardless of the choice of a color for $b c$, which counts in the sums of both, $b$ and $c)$. All together, we are left with at least $\left\lceil\frac{7 \Delta-4}{2}\right\rceil-3(\Delta-1)=\left\lceil\frac{\Delta+2}{2}\right\rceil$ safe choices of colors for $a b$. Analogously, we have also at least $\left\lceil\frac{\Delta+2}{2}\right\rceil$ available colors for $b c$ (with $a b$ not painted yet).

All we have to do now is to choose the colors for $a b$ and $b c$ from these lists of lengths at least $\left\lceil\frac{\Delta+2}{2}\right\rceil$ so that these colors are different, and so that the sum at $b$ is different from at most $\Delta-2$ (already determined) sums at $b_{1}, \ldots, b_{q}$. To show that this is always possible, it is enough to note that for any two lists of numbers $X, Y$, each of size $s$, there exist (at least) $2 s-3$ pairs $\left(x_{i}, y_{i}\right) \in X \times Y$ with $x_{i} \neq y_{i}, i=$ $1, \ldots, 2 s-3$, for which all the sums $x_{i}+y_{i}$ are pairwise distinct (in particular, for $s=\left\lceil\frac{\Delta+2}{2}\right\rceil$, we have $\left.2 s-3 \geq(\Delta-2)+1\right)$. Indeed, these are e.g. the pairs from the set $(\{x\} \times(Y \backslash\{x\})) \cup((X \backslash\{x, y\}) \times\{y\})$, where $x=\min X$ and $y=\max Y$.

It is easy to verify that the obtained coloring fulfils our requirements (even if there were some isolated edges or vertices among the components of $H$ ). Finally, if some of the sets $N_{H}(a), N_{H}(b), N_{H}(c)$ are empty, the proof is analogous.

This theorem implies immediately the following result.

Theorem 8 If $G$ is a connected graph with maximum degree $\Delta \geq 2$, then

$$
\operatorname{ndi}(G) \leq\left\lceil\frac{7 \Delta-4}{2}\right\rceil .
$$

The last theorem is weaker than the results of of Akbari et al. [1] and Hatami [6] for graphs with large maximum degree. 


\section{Trees}

Theorem 9 Let $T$ be a tree of order $n \geq 3$ and maximum degree $\Delta$. Then $\operatorname{ndi}_{\Sigma}(T)=$ $\Delta$ if the set of vertices of degree $\Delta$ is a stable one, and $\operatorname{ndi}_{\Sigma}(T)=\Delta+1$ otherwise.

Proof We proceed by induction on $n$. Observe that the theorem is trivial if $T$ is a star $K_{1, n-1}$ or a path, hence, in particular, for $n=3$. Furthermore, the incident edges to two adjacent vertices of maximum degree in $T$ cannot be colored with $\Delta$ colors, so if the set of vertices of degree $\Delta$ is not a stable one, then $\operatorname{ndi}_{\Sigma}(T) \geq \Delta+1$. Suppose our assertion is true for all trees of order at most $n-1$ and let $T$ be a tree of order $n$. We may assume that $T$ is isomorphic neither to $K_{1, n-1}$ nor to a path.

Let $x$ be an endvertex of a longest path in $T$, chosen such that if $T$ has only one vertex of degree $\Delta$, it is not the only neighbor $y$ of $x$.

Let $T^{\prime}$ denote the tree $T \backslash\{x\}$. By the choice of $x, y$ has only one neighbor, say $z$, of degree $\geq 2$, and for any vertex $t \in V\left(T^{\prime}\right)$ its degree $d_{T^{\prime}}(t)=d^{\prime}(t)$ in $T^{\prime}$ is the same as in $T$, except for $t=y$ for which $d^{\prime}(y)=d(y)-1$. Thus $\Delta\left(T^{\prime}\right)=\Delta \geq 3$.

By induction hypothesis, there is a proper nsd [k]-edge coloring $v$ of $T^{\prime}$ with $k=\operatorname{ndi}_{\Sigma}\left(T^{\prime}\right)$. Put for $t \in V\left(T^{\prime}\right), w(t)=\sum_{u \in N_{T^{\prime}}(t)} v(t u)$ and let $A=\left[\operatorname{ndi}_{\Sigma}\left(T^{\prime}\right)\right] \backslash$ $\left\{v(y t) \mid y t \in E\left(T^{\prime}\right)\right\}(\neq \emptyset)$.

If there is in $T^{\prime}$ a neighbor $t$ of $y$ distinct from $z$ then $d^{\prime}(t)=1$ and $w(t)<w(y)$, so in order to extend $v$ into an nsd $[\Delta]$-edge coloring (or an nsd $[\Delta+1]$-edge coloring) of $T$ it is sufficient either to choose a value $v(x y)=a \in A$ in such a way that $w(y)+a \neq w(z)$ (if we have to prove that $\operatorname{ndi}_{\Sigma}(T)=\operatorname{ndi}_{\Sigma}\left(T^{\prime}\right)$ ) or to color $x y$ with the color $\Delta+1$ (if we have to prove that $\operatorname{ndi}_{\Sigma}(T)=\Delta+1=\operatorname{ndi}_{\Sigma}\left(T^{\prime}\right)+1$ ).

Case $1 d^{\prime}(y) \leq \Delta-2$ or $\left(d^{\prime}(y)=\Delta-1\right.$ and $\left.\operatorname{ndi}_{\Sigma}\left(T^{\prime}\right)=\Delta+1\right)$. Then $|A| \geq 2$ and there is at least one $a \in A$ such that $w(y)+a \neq w(z)$. Putting $v(x y)=a$ we get the desired coloring of $T$.

Case $2 d^{\prime}(y)=\Delta-1$ and $\operatorname{ndi}_{\Sigma}\left(T^{\prime}\right)=\Delta$. Then $A$ contains only one element $a, 1 \leq a \leq \Delta$. If $d^{\prime}(z)<\Delta$, then $w(z)<1+2+\cdots+\Delta=a+w(y)$ and we may put $v(x y)=a$ to obtain an nsd $[\Delta]$-edge coloring of $T$. The case $d^{\prime}(z)=\Delta$ is a special one, since we have to prove that $\operatorname{ndi}_{\Sigma}(T)=\Delta+1\left(=\operatorname{ndi}_{\Sigma}\left(T^{\prime}\right)+1\right)$. In this case, $w(z)=1+2+\cdots+\Delta<w(y)+(\Delta+1)$ and we may put $v(x y)=\Delta+1$ to get the desired coloring of $T$.

\section{Regular Graphs}

Recall that a Latin square of order $n$ is an $n \times n$ matrix $L(n)=\left(c_{i j}\right)$ such that each entry belongs to the set $[n]$ and each integer of $[n]$ occurs in each row and each column exactly once. A transversal Latin square of order $n$ is a Latin square of order $n$ such that every element of $[n]$ appears exactly once in the main diagonal. It is well known (cf. [4]) that for every $n \geq 3$ there exists a transversal Latin squares of order $n$.

Proposition 10 For $n \geq 3, \operatorname{ndi}_{\Sigma}\left(K_{n, n}^{\prime}\right)=n$.

Proof Let $L(n)=\left(c_{i j}\right)$ be a transversal Latin square of order $n$. Let $X=\left\{x_{i}, \ldots, x_{n}\right\}$ and $Y=\left\{y_{1}, \ldots, y_{n}\right\}$ be the two sets of the bipartition of $K_{n, n}^{\prime}$ and assume $x_{i} y_{i} \notin$ 
$E\left(K_{n, n}^{\prime}\right), i=1, \ldots, n$. Color $x_{i} y_{j}$ with $c_{i j}, i, j=1, \ldots, n, i \neq j$. It is obvious that this is a proper nsd $[n]$-edge coloring and there is no nsd $[n-1]$-edge coloring of $K_{n, n}^{\prime}$.

If $G=(V, E)$ and $H=(W, F)$ are two graphs, the Cartesian product of graphs $G$ and $H$ is defined to be the graph $G \times H$ whose vertex set is $V \times W$ and whose two vertices $(x, y)$ and $\left(x^{\prime}, y^{\prime}\right)$ are adjacent if and only if either $x=x^{\prime}$ and $y y^{\prime} \in F$, or $y=y^{\prime}$ and $x x^{\prime} \in E$.

Theorem 11 Let $G$ be a d-regular graph with $\operatorname{ndi}_{\Sigma}(G)=d+1$ and $H$ a d'-regular bipartite graph such that there exists a partition of $E(H)$ into $d^{\prime}$ perfect matchings $\left(E_{i}\right)_{1 \leq i \leq d^{\prime}}$ (H belongs to the Vizing class 1$)$. Then $\operatorname{ndi}_{\Sigma}(G \times H)=d+d^{\prime}+1$.

Proof Let $v$ be an nsd $[d+1]$-edge coloring of $G$ (note that $\operatorname{ndi}_{\Sigma}(G) \geq d+1$ for any $d$-regular graph $G$ ). For any $x \in V(G)$ there exists one and only one value $v^{\prime}(x) \in[d+1]$ lacking in the set of edges incident to $x$. Let $s$ be a permutation of the set $[d+1]$ without fixed point. Then for all $x$ in $V(G) s\left(v^{\prime}(x)\right) \neq v^{\prime}(x)$. If $X, Y$ are the two classes of the bipartition of $H$, put the coloring $v$ on the edges of all the copies of $G$ in $G \times H$ indexed by $X$, the coloring $s v$ on the other ones, and put the color $d+1+i$ on the edges corresponding to the matching $E_{i}, i=1, \ldots, d^{\prime}$. It is clear that this is an nsd $\left[d+d^{\prime}+1\right]$-edge coloring of $G \times H$ (notice that $G \times H$ is $\left(d+d^{\prime}\right)$-regular).

Corollary 12 If $G$ is a d-regular with $\operatorname{ndi}_{\Sigma}(G)=d+1$, then $\operatorname{ndi}_{\Sigma}\left(G \times K_{2}\right)=d+2$.

Observe that $\operatorname{ndi}_{\Sigma}\left(Q_{3}\right)=4$, where $Q_{n}$ denotes the $n$-dimensional hypercube. Thus, the following corollary is true.

Corollary 13 For the hypercube $Q_{n}$, $\operatorname{ndi}_{\Sigma}\left(Q_{n}\right)=n+1$ if $n \geq 3$.

\section{Graphs with Maximum Degree at Most 3}

\subsection{Cubic Hamiltonian Graphs}

By Theorem 7, $\operatorname{ndi}_{\Sigma}(G) \leq 9$ for 3-regular graphs. On the other hand, by [3], $\operatorname{ndi}(G) \leq$ 5 for these graphs. Using the concept of maximal (for a given $n$ ) Sidon sets, $S_{n}$ (that is any maximal size subset of $[n]$ whose each pair of distinct elements has a unique sum, i.e., $a+b \neq c+d$ for any $a, b, c, d \in S_{n}, a \neq b, c \neq d,\{a, b\} \neq\{c, d\}$ ), we immediately obtain the following improvement of our bound for cubic graphs.

Theorem 14 If $G$ is a 3-regular graph, then $\operatorname{ndi}_{\Sigma}(G) \leq 8$.

Proof Given any proper 5-edge coloring distinguishing the neighbors by sets, whose existence for cubic graphs was proven in [3], it is enough to use the colors from the set $S_{8}\left(S_{8}=\{1,2,3,5,8\}\right)$ instead of the original ones. The obtained proper [8]-coloring $v$ of $G$ is nsd, since $S(x) \cap S(y)=\{v(x y)\}$ and $S(x) \backslash\{v(x y)\} \neq S(y) \backslash\{v(x y)\}$ whenever vertices $x, y$ are adjacent in $G$. 
Moreover, the reasoning used in [3] to prove that $\operatorname{ndi}(G) \leq 5$ for all hamiltonian 3-regular graphs is also useful in our case, after a small adjustment of its final part. Here our concept is based on an adequate replacement of the colors from the Klein group mentioned below with the ones from a Sidon set $S_{5}$ (that is either $\{1,3,4,5\}$ or $\{1,2,3,5\})$.

Theorem 15 If $G$ is a 3-regular hamiltonian graph, then $\operatorname{ndi}_{\Sigma}(G) \leq 5$.

Proof Let $\{0, a, b, c\}$ be the elements of the Klein group $K=\mathbb{Z}_{2} \times \mathbb{Z}_{2}$, where $x+x=0$ for all $x$ and $x+y=z$ whenever $\{x, y, z\}=\{a, b, c\}$. We shall first find a proper 5-edge coloring distinguishing the neighbors by sets using colors from the set $K \cup\{2\}$. All colors from $K$ will be then replaced with the ones from a maximal Sidon set $A_{5}=\{1,3,4,5\}$.

Let $C=x_{1} \ldots x_{n}$ be a hamiltonian cycle of $G$ and let $I$ be the remaining 1-factor of this graph. Since $\operatorname{ndi}_{\Sigma}\left(K_{4}\right)=5$ by Proposition 4, we may assume by Brooks' theorem that $f: V \rightarrow\{a, b, c\}$ is a proper vertex coloring of $G$. We may additionally assume that each of the three colors appears at least once in this coloring (since otherwise we could simply recolor one of the vertices). Let $S=\sum_{i=1}^{n} f\left(x_{i}\right) \in K$.

If $S=0$ then label $x_{n} x_{1}$ with 0 and inductively label $x_{i} x_{i+1}$ for $i=1, \ldots, n-1$ so that $f\left(x_{i}\right)$ is the sum (in the group $K$ ) of the colors on $x_{i-1} x_{i}$ and $x_{i} x_{i+1}$. Equivalently, the color on $x_{i} x_{i+1}$ is the sum of the colors on $x_{i-1} x_{i}$ and $f\left(x_{i}\right)$. Then $f\left(x_{n}\right)$ is the sum of the colors on $x_{n} x_{1}$ and $x_{n-1} x_{n}$. Thus the above labeling works also for $i=n$ provided that indices are taken modulo $n$.

Color the 1-factor $I$ with the color 2. Each vertex $x$ sees (in its color set) the color 2 and two colors from $K$ summing up to $f(x)$. Since $f(x) \neq 0$, these two colors from $K$ are distinct (hence the edge coloring is proper), and since $f(x) \neq f(y)$ for any two adjacent vertices $x$ and $y$, the color sets at $x$ and $y$ must be distinct. Now it is enough to (arbitrarily) replace the colors of the edges from $K$ with the ones from the Sidon set $A_{5}$. After such a substitution, each pair of neighbors $x$ and $y$ must be distinguished by sums, since each of them sees the color 2 , while the pairs of the remaining colors are distinct.

Now suppose that $S \neq 0$. Without loss of generality we may assume $S=c$. Pick any vertex $x_{i}$ with $f\left(x_{i}\right)=c$. Let $x_{i} x_{j} \in I$. Then $f\left(x_{j}\right)$ equals either $a$ or $b$. Recolor $x_{j}$ with $b$ or $a$, respectively. Now $S=0$ and we can color as above the edges of the hamiltonian cycle $C$ with the elements of $K$. Coloring $I$ with 2 yields a proper edge coloring that distinguishes all the adjacent vertices, with a possible exception for $x_{j}$, by sets. Since $f\left(x_{i}\right) \neq f\left(x_{j}\right)$, the pair of colors from $K$ meeting $x_{i}$ cannot be disjoint from the pair that meets $x_{j}$. Hence there must be some color $d \in K$ missing on the edges incident to $x_{i}$ or $x_{j}$ and at least one, say $e$, (from $K$ ) that meets both, $x_{i}$ and $x_{j}$. First we recolor the edge $x_{i} x_{j}$ with this missing color $d$ (hence the edge coloring remains proper). Then we replace the colors of the edges from $K$ by the ones from $A_{5}$, this time more carefully, namely, so that $d=5$ and $e=4$. Analogously as above, all the vertices are distinguished from their neighbors by sums, except for some possible conflicts in the neighborhoods of $x_{i}$ and $x_{j}$. Note then that since we had $f\left(x_{i}\right) \neq f\left(x_{j}\right)$, the sum at $x_{i}$ must be distinct from the one at $x_{j}$. Consider then $x_{i}$ and its two neighbors $x_{i-1}, x_{i+1}$ (for $x_{j}$ and $x_{j-1}, x_{j+1}$ the reasoning is the same). Then there are only two possibilities. First, when at $x_{i}$ we have the sum $5+4+3=12$, 
while $x_{i-1}, x_{i+1}$ both see the color 2 , hence their sums are smaller than 12 . Second, when we have $5+4+1=10$ at $x_{i}$, while the sums for $x_{i-1}$ and $x_{i+1}$ can only be of the form either $4+2+t \neq 10$ (since the edge coloring is proper) or $s+2+1<10$. In either case we obtain a proper neighbor sum distinguishing [5]-edge coloring of $G$.

\subsection{A Class of Graphs with Maximum Degree Three}

It is an easy exercise to check that $\operatorname{ndi}_{\Sigma}(G)=4$, where $G$ is the Petersen graph. For other nonhamiltonian graphs of maximum degree 3 we are able to prove the following theorem.

Theorem 16 Let $G$ be a connected simple graph with maximum degree $\Delta=3$, such that the set of vertices of degree 3 is independent. Then $\operatorname{ndi}_{\Sigma}(G)=3$ or 4 .

The proof of this theorem is long and will be published elsewhere.

Open Access This article is distributed under the terms of the Creative Commons Attribution License which permits any use, distribution, and reproduction in any medium, provided the original author(s) and the source are credited.

\section{References}

1. Akbari, S., Bidkhori, H., Nosrati, N.: r-Strong edge colorings of graphs. Discrete. Math. 306, 30053010 (2006)

2. Bondy, J.A., Murty, U.S.R.: Graph Theory with Applications. Macmillan, London (1976)

3. Balister, PN., Győri, E., Lehel, L., Schelp, R.H.: Adjacent vertex distinguishing edge-colorings. SIAM. J. Discrete. Math. 21, 237-250 (2007)

4. Dénes, J., Keedwell, A.D.: Transversal and Complete Mappings, In: Latin Squares. New Developments in the Theory and Applications, Elsevier, Amsterdam (1991)

5. Edwards, K., Horňák, M., Woźniak, M.: On the neighbour-distinguishing index of a graph. Graphs. Combin. 22, 341-350 (2006)

6. Hatami, H.: is a bound on the adjacent vertex distinguishing edge chromatic number. J. Combin. Theory. Ser B. 95, 246-256 (2005)

7. Mahéo, M., Saclé, J-F.: Some results on $(\Sigma, p, g)$-valuation of connected graphs, Rapport de Recherche 1497, Université de Paris-Sud, Centre d'Orsay (2008)

8. Zhang, Z., Liu, L., Wang, J.: Adjacent strong edge coloring of graph. Appl. Math. Lett. 15, 623626 (2005) 